\title{
Impact Forces Occurring in a Forced Damped Multi-Body System with Clearances
}

\author{
Guillaume Ricciardi \\ CEA, Alternative Energies and Atomic Energy Commission, France
}

The purpose of this paper is to study the effect of clearance and other parameters on the maximal impact forces likely to occur in a forced multi-body system. Numerical simulations are performed for a large range of parameters. The numerical results showed three regions. In the first one, there is no contact between the bodies. The second one, defined as the smooth region, is characterized by regular evolution with a concentration of the impact forces at the boundaries. In the last one, where the most important impact forces are met, the system shows chaotic behaviours and coincides with the lower values of clearance. Theoretical upper bounds for the maximal forces are proposed and compared to numerical results. In the smooth region, the criterion is respected, but in the chaotic region, some discrepancies are observed. Nevertheless, the criterion gives a good estimation. The dimensionless analysis showed that the maximal impact force is proportional to the mass, the acceleration, the stiffness ratio and the inverse of the damping ratio.

Keywords: multi-body, impact, clearance, damping

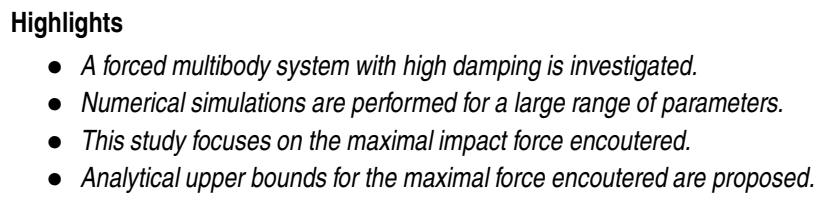

- Numerical simulations are performed for a large range of parameters.

- This study focuses on the maximal impact force encoutered.

- Analytical upper bounds for the maximal force encoutered are proposed.

\section{INTRODUCTION}

Mechanical systems involving several components separated by clearances can be encountered in many engineering fields as energy [1], automobile [2] or space industry [3]. When subjected to an external excitation, impacts occur between the components and the force resulting from these impacts may cause damages. The impact forces strongly depend on the clearance [4] and increase with contact stiffness [5] and the number of clearances [6]. The nonlinear system gives rise to chaotic behaviours with a large variety of transitions between chaotic and periodic behaviours using clearance as a parameter [7]. This complexity is illustrated by [8] and [9]: they propose maps using clearance and excitation frequency as parameters showing the number of impacts within a period: it appears that the more complex behaviours are obtained for lower values of clearance. Some have proposed an analytical analysis of bifurcations [10,11] and showed discontinuity in bifurcation maps. Transitions between chaotic and periodic solutions have been widely studied on many nonlinear mechanical systems, such as pendulum [12], tank filled with water [13], disk brake [14], or cracked rotor [15].

This study is applied to a liquid metal fast breeder nuclear reactor core. This core is made of clamped-free slender structures immersed in a liquid as described in [16]. In case of a seismic event the impact force generated by the contact between bodies can be damaging; therefore, knowing the maximal force occurring in case of an earthquake is fundamental to making a proper design. The purpose of this paper is to study the effect of clearance and other parameters on the maximal impact forces likely to occur in a forced multi-body system. A dimensionless expression of the dynamic equation is proposed, and numerical simulations are performed for a large range of parameters, assuming that the slender structures only vibrate on their first natural bending mode.

\section{METHODOLOGY}

Let us consider the simple system composed of $\mathrm{Nm}$ bodies of mass $M$ attached to the ground by a spring $K$ and a viscous damping $C$, the displacement of the ground $u_{e}$ is imposed as in a seismic event, and the displacement of each body $i$ is noted $u_{i}$ (Fig. 1). The bodies are separated by a clearance $e$, and when a contact is detected impact forces, $F_{l i}$ and $F_{r i}$ are imposed either on the left or the right of the body. The equation of motion of each body $i$ is given by:

$M \frac{\partial^{2} u_{d i}}{\partial t^{2}}+C \frac{\partial u_{d i}}{\partial t}+K u_{d i}=-M \frac{\partial^{2} u_{e}}{\partial t^{2}}+F_{l i}+F_{r i}$ 
where $u_{d i}$ is the relative displacement:

$$
u_{d i}=u_{i}-u_{e}
$$

The impact forces are modelled by linear springs $K_{c}$ when the contact is detected to account for the stiffness contact that can be different from the stiffness of the system. Different models can be found to account for the contact between bodies, by the continuity of the displacements or the velocities when the contact is detected or with nonlinear stiffness given by the Hertz theory. In the present study, a linear spring is chosen to account for the local deformation of the slender body at the contact location which is much higher than the bending stiffness $K$. This is a very critical parameter that can completely change the behaviour of the structure.

$$
\begin{gathered}
\forall i \in[2: N m], F_{l i}=\left\{\begin{array}{l}
\frac{K_{c}}{2}\left(u_{d i-1}-u_{d i}-e\right) \\
\text { if }\left(u_{d i-1}-u_{d i}-e\right)>0, \\
0 \\
\text { if }\left(u_{d i-1}-u_{d i}-e\right)<0,
\end{array}\right. \\
F_{r i}=\left\{\begin{array}{l}
-\frac{K_{c}}{2}\left(u_{d i}-u_{d i+1}-e\right) \\
\text { if }\left(u_{d i}-u_{d i+1}-e\right)>0, \\
0 \\
\text { if }\left(u_{d i}-u_{d i+1}-e\right)<0,
\end{array}\right.
\end{gathered}
$$

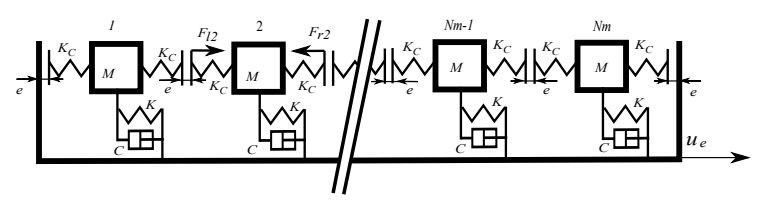

Fig. 1. Multi-body system with clearances

The acceleration of the ground is assumed harmonic at the frequency $f$ and for an amplitude of $a_{0}$ :

$$
\frac{\partial^{2} u_{e}}{\partial t^{2}}=a_{0} \cos (2 \pi f t)
$$

In the following, the dimensionless equations are established based on the following dimensionless quantities:

$$
f_{0}=\frac{1}{2 \pi} \sqrt{\frac{K}{M}}
$$

$$
\begin{aligned}
f_{r} & =\frac{f}{f_{0}} \\
f_{c} & =\frac{K_{c}}{K} \\
\varepsilon & =\frac{C}{2 \sqrt{K M}}, \\
t_{r} & =f_{0} t \\
e_{r} & =\frac{4 \pi^{2} f_{0}^{2} e}{a_{0}}, \\
u_{r i} & =\frac{4 \pi^{2} f_{0}^{2} u_{d i}}{a_{0}}, \\
v_{r i} & =\frac{\partial u_{r i}}{\partial t_{r}}, \\
F_{l r i} & =\frac{F_{l i}}{M a_{0} \sqrt{f_{c}}} \\
F_{r r i} & =\frac{F_{r i}}{M a_{0} \sqrt{f_{c}}} .
\end{aligned}
$$

Thus the equations of the system become:

$$
\begin{aligned}
& \frac{1}{4 \pi^{2}} \frac{\partial^{2} u_{r i}}{\partial t_{r}^{2}}+\frac{\varepsilon}{\pi} \frac{\partial u_{r i}}{\partial t_{r}}+u_{r i}=-\cos \left(2 \pi f_{r} t_{r}\right) \\
& +\sqrt{f_{c}} F_{l r i}+\sqrt{f_{c}} F_{r r i}, \\
& \forall i \in[2: N m], \\
& F_{l r i}=\left\{\begin{array}{l}
\frac{\sqrt{f_{c}}}{2}\left(u_{r i-1}-u_{r i}-e_{r}\right) \\
\text { if }\left(u_{r i-1}-u_{r i}-e_{r}\right)> \\
0, \\
0 \\
\text { if }\left(u_{r i-1}-u_{r i}-e_{r}\right)<0,
\end{array}\right. \\
& \forall i \in[1: N m-1], \\
& F_{r r i}=\left\{\begin{array}{l}
-\frac{\sqrt{f_{c}}}{2}\left(u_{r i}-u_{r i+1}-e_{r}\right) \\
\text { if }\left(u_{r i}-u_{r i+1}-e_{r}\right)>0 \\
0 \\
\text { if }\left(u_{r i}-u_{r i+1}-e_{r}\right)<0
\end{array}\right.
\end{aligned}
$$

The response of the system is totally defined by the stiffness ratio $f_{c}$, the number of bodies $\mathrm{Nm}$, the damping ratio $\varepsilon$, the reduced clearance $e_{r}$ and the reduced frequency imposed to the system $f_{r}$.

To observe the dependency of impact forces on dimensionless parameters, a parametric study is performed numerically (Table 1). The range studied corresponds to the different situations and configurations encountered in the seismic response of reactor cores. For each set of parameters $\left(f_{c}, N m, \varepsilon, e_{r}\right.$, 
and $f_{r}$ ), a temporal numerical simulation is performed with an explicit time scheme for 1000 periods. For the present study over 430,000 simulations have been performed. For each simulation we note $F_{m}$ the maximal impact force in time and space:

$$
F_{m}=\max \left(F_{r r i}\left(t_{r j}\right), F_{l r i}\left(t_{r j}\right)\right)_{\forall(i, j)} .
$$

In the following, most of the results shown are accounting for a damping ratio of $50 \%$ which will be the default value without indication. In this paper, only high values of damping are considered because while it makes the simulations simpler because the harmonic regime is reached faster, these high values are commonly encountered in the industry especially with structures surrounded by fluid [17].

Table 1. Parameters range used for numerical simulations

\begin{tabular}{ccccc}
\hline$f_{c}$ & $N m$ & $\varepsilon$ & $e_{r}$ & $f_{r}$ \\
\hline 1 to 2000 & 1 to 20 & 10 to 50 & 1 to 6 & 1 to 5 \\
\hline
\end{tabular}

\section{GENERAL BEHAVIOUR}

For each couple of $\mathrm{Nm}$ and $f_{c}$, Fig. 2 shows the maximal reduced impact force $F_{m}$ as a function of $f_{r}$ and $e_{r}$. Different behaviours can be observed, but three zones can be identified. The first one corresponds to $F_{m}$ $=0$, which means that there is no impact either because the reduced gap is too high or because the frequency is too far from resonance. The second zone corresponds to $e_{r}>0.3$ and will be referred to as the smooth zone. In that zone the force $F_{m}$ shows a regular evolution with a maximum around $f_{r}=1$ close to the natural frequency of the system; this zone seems to show an identical trend for every $\left(\mathrm{Nm}, f_{\mathrm{c}}\right)$ couple. The third zone, referred to as a chaotic zone for $e_{r}<0.3$, shows irregular evolution and can be very different depending on the $\left(N m, f_{c}\right)$ couple. This zone shows the highest values of $F_{m}$.

The limits of the noimpact zone can be easily estimated considering the response of a single body with no interaction, of which the modulus is given by:

$$
\left|u_{0}\right|=\frac{1}{\sqrt{\left(1-f_{r}^{2}\right)^{2}+4 \varepsilon^{2} f_{r}^{2}}} .
$$

It can be seen that the previous equation (red curve) fits the smallest values of $F_{m}$ in Fig. 3 . (a)

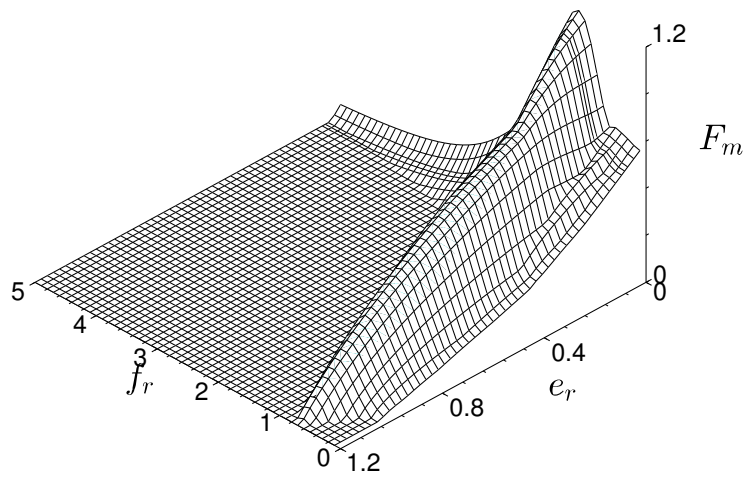

(b)

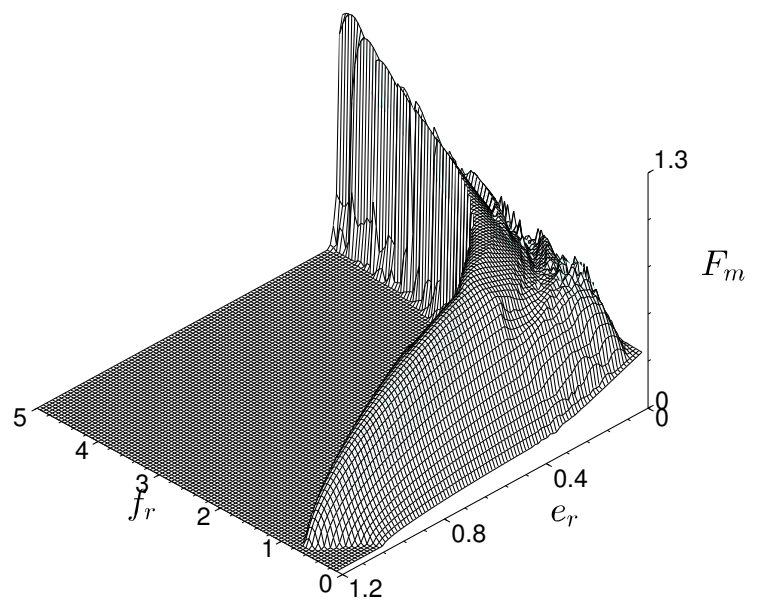

Fig. 2. Maximal reduced impact force as function of $e_{r}$ and $f_{r}$, for $\mathrm{Nm}=$ 3 and $f_{c}=3(a)$, for $N m=3$ and $f_{c}=100(b)$

Figs. 4 to 7 show the evolution of $F_{m}$ as a function of the reduced clearance for two reduced frequencies and various $\left(\mathrm{Nm}, f_{c}\right)$ couples. In the smooth region, close to the natural frequency at $f_{r}=1, F_{m}$ shows an asymptotic behaviour as $\mathrm{Nm}$ and $f_{c}$ increase. At lower frequencies the behaviour is different, $F_{m}$ does not depend on the number of bodies $\mathrm{Nm}$, but it decreases as the stiffness ratio $f_{c}$ increases. For lower $e_{r}$, the only clear trend is for lower frequencies where $F_{m}$ increases with $N m$ whereas it decreases when $f_{c}$ increases. For other frequencies, one can see strong fluctuations. 
The chaotic behaviour is more important for higher values of $f_{c}$.

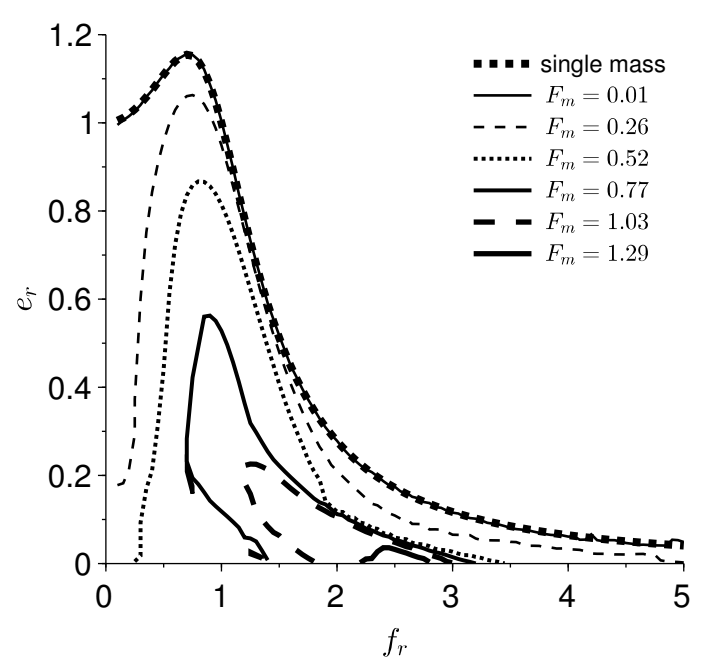

Fig. 3. Iso $F_{m}$ curves for $\mathrm{Nm}=5$ and $f_{c}=60$, with the theoretical response of a single mass (thick dotted curve)

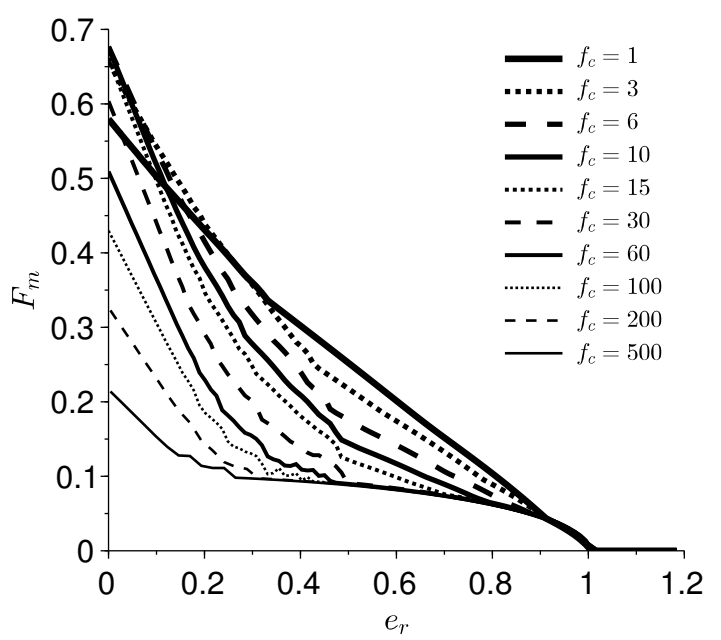

Fig. 4. Maximal reduced impact force as a function of $e_{r}$ for $\mathrm{Nm}=5$ and various $f_{c}$ for $f_{r}=0.1$

Fig. 8 shows the evolution of the spatial distribution of $F_{m}$ close to the natural frequency as a function of $e_{r}$. It can be seen that in the smooth region $\left(e_{r}>0.3\right)$ only a few masses at the extremities are in contact. As $e_{r}$ decreases, the system goes in the chaotic region, and the number of bodies in contact increases

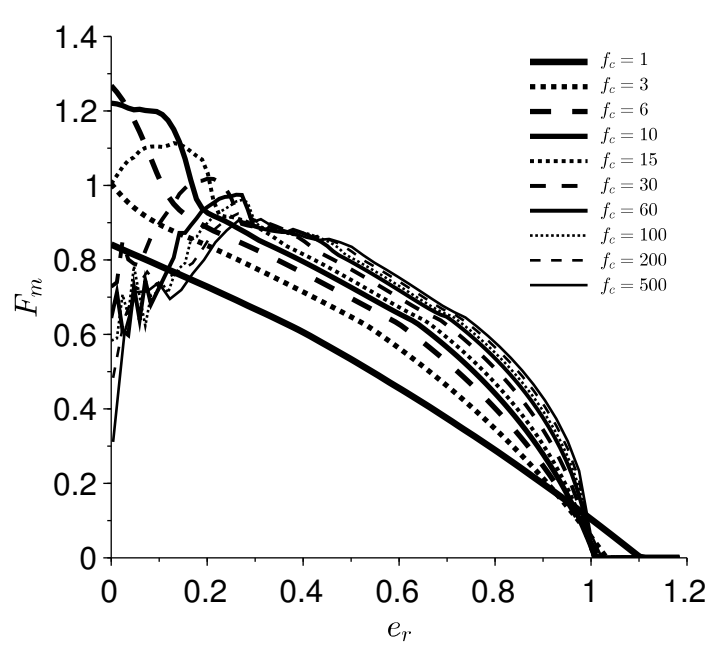

Fig. 5. Maximal reduced impact force as a function of $e_{r}$ for $\mathrm{Nm}=5$ and various $f_{c}$ for $f_{r}=1$

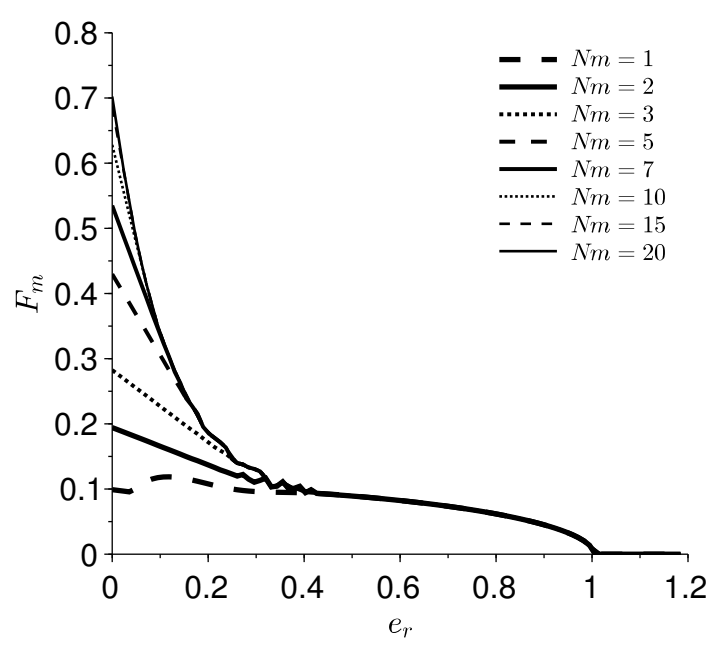

Fig. 6. Maximal reduced impact force as a function of $e_{r}$ for $f_{c}=100$ and various $\mathrm{Nm}$ for $f_{r}=0.1$

to reach a state in which all bodies are in contact, and comparable values of impact forces are seen.

The evolution of $F_{m}$ as a function of frequency (Fig. 9) shows the difference between a non-chaotic $\left(f_{c}\right.$ $=10)$ and chaotic behaviour $\left(f_{c}=100\right)$. In the first one, the impact forces are always located at the extremities with a regular spatial evolution, whereas in the second one the maximal values can be located anywhere, and disturbed spatial evolution is shown. 


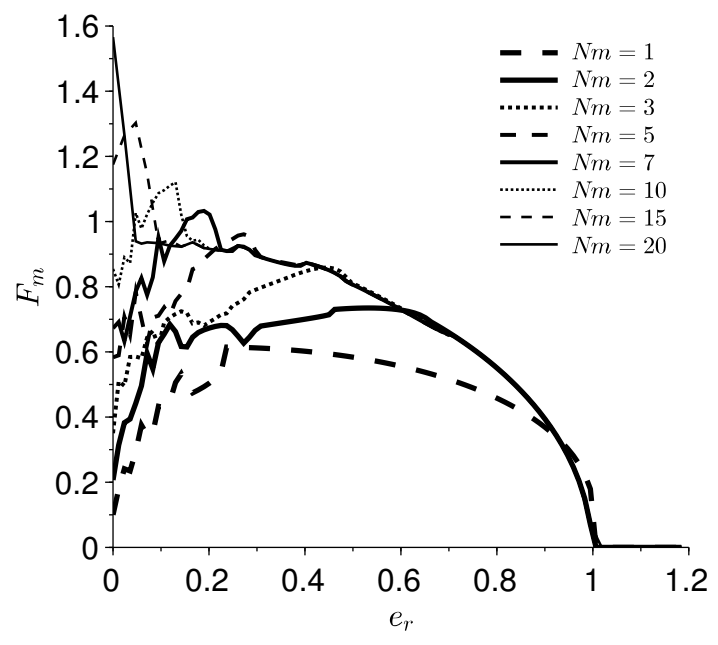

Fig. 7. Maximal reduced impact force as a function of $e_{r}$ for $f_{c}=100$ and various $\mathrm{Nm}$ for $f_{r}=1$

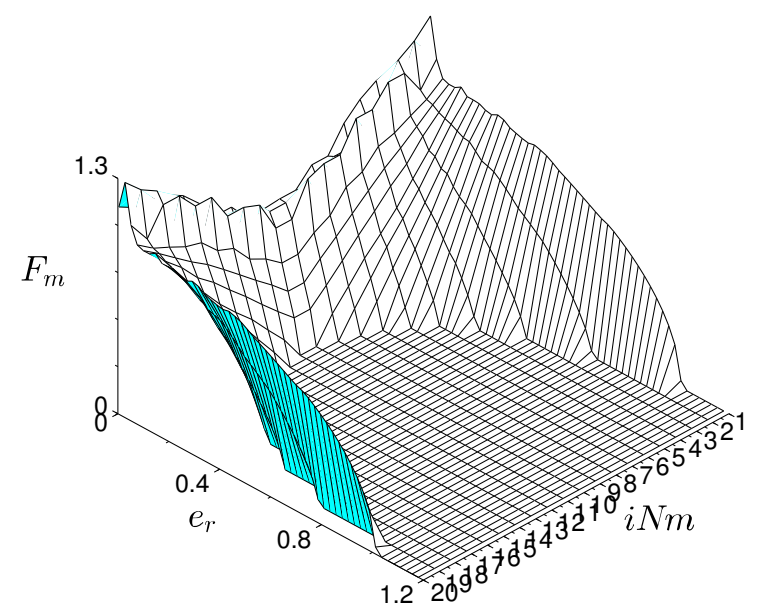

Fig. 8. Maximal reduced impact force spatial distribution as function of $e_{r}$ for $\mathrm{Nm}=20, f_{r}=1$ and $f_{c}=200$

\section{SMOOTH REGION}

In the smooth region, the maximal impact force is obtained at the natural frequency $\left(f_{r}=1\right)$ with a small shift toward higher frequencies when $f_{c}$ increases for lower values of $e_{r}$ (Fig. 10). At a given $f_{c}, F_{m}$ quickly converges as $\mathrm{Nm}$ increases, for $e_{r}=0.24$ the results do not change after $N m=5$, and for higher values of $e_{r}$, the asymptotic value is reached for $N m=2$ (Fig. 11).

(a)

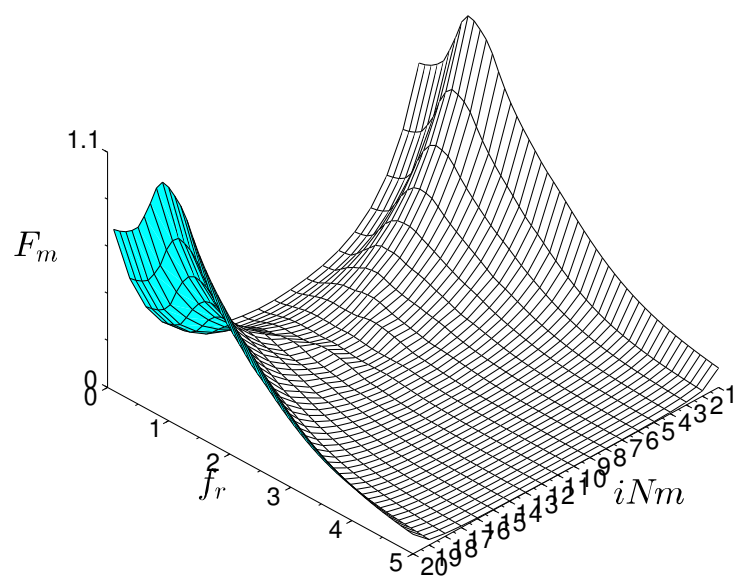

(b)

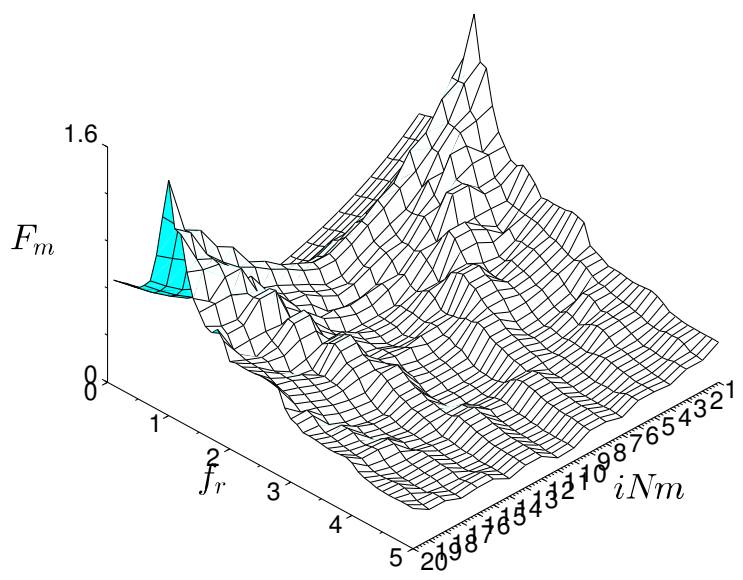

Fig. 9. Maximal reduced impact force spatial distribution as function of $f_{r}$ for $\mathrm{Nm}=20, e_{r}=0$ and $f_{c}=1$ (a), $f_{c}=100$ (b)

When only a few bodies show contact the number of bodies in contact does not depend on the total number of contact and explain why the asymptotic value is quickly reached.

Fig. 12 shows the influence of damping ratio on the maximal impact force; it can be seen that as this ratio decreases, the force increases and that the transitions between chaotic and smooth regions are 


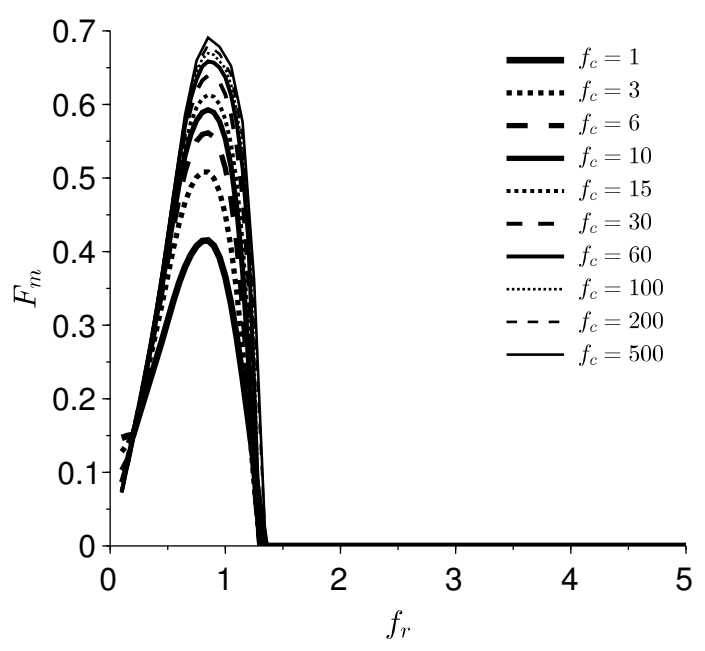

Fig. 10. Maximal reduced impact force as function of $f_{r}$ for $\mathrm{Nm}=5$ and various $f_{c}$ for $e_{r}=0.71$

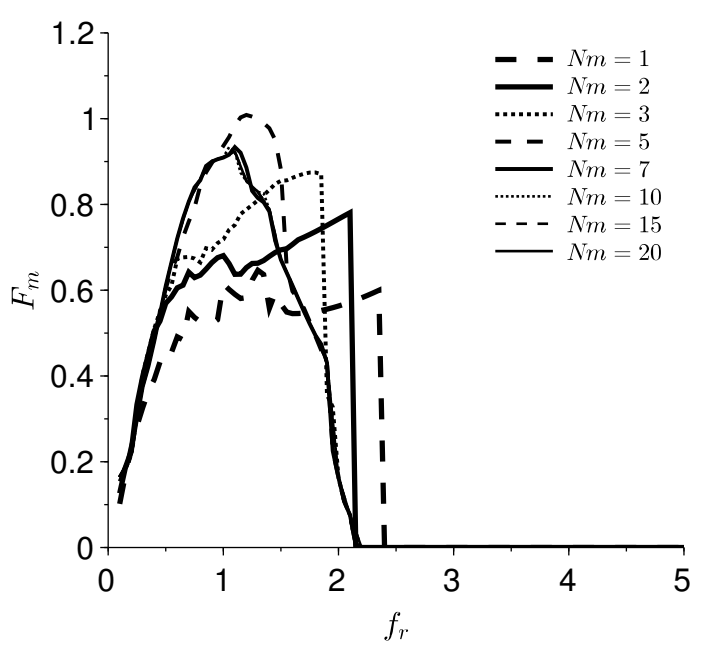

Fig. 11. Maximal reduced impact force as function of $f_{r}$ for $f_{c}=100$ and various $\mathrm{Nm}$ for $e_{r}=0.24$

shifted toward higher values of $e_{r}$. This is an expected results, since the damping dissipates energy.

Based on the latter observations, one could assume that the maximal impact force in the smooth region is given by the oscillation of a mass at the extremities in contact with the wall.

This system has a natural frequency $f_{1}$ :

$$
f_{1}=\sqrt{1+f_{c}}
$$

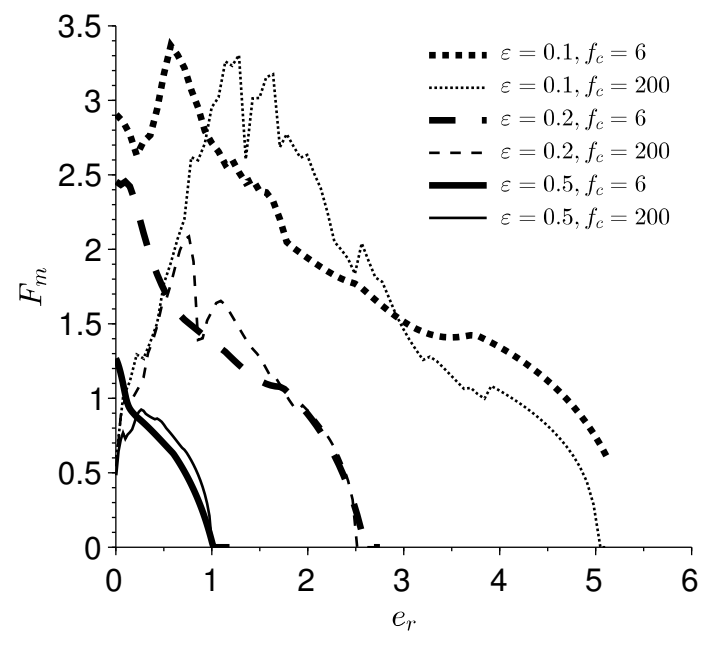

Fig. 12. Maximal reduced impact force as a function of $e_{r}$ at $f_{r}=1$ for various $\varepsilon$, and for $N m=5$ and various $f_{c}$

The maximum amplitude $u_{m}$ is given by:

$$
u_{m}=\frac{1}{2 \varepsilon \sqrt{1+f_{c}-\varepsilon^{2}}} .
$$

The force is given by the compression of the spring $K_{c}$, in the dimensionless form it gives for the maximal force:

$$
\begin{aligned}
F_{m} & =\sqrt{f_{c}} u_{m}, \\
& =\frac{1}{2 \varepsilon} \sqrt{\frac{f_{c}}{1+f_{c}-\varepsilon^{2}}} .
\end{aligned}
$$

Thus, the maximal force $F_{m}$ depends on the stiffness ratio $f_{c}$ and the damping ratio $\varepsilon$. One can extract from the previous equation an upper bound depending only on $\varepsilon$ :

$$
F_{m}<\frac{1}{2 \varepsilon}
$$

The observation of the numerical results shows that the criterion (25) is always verified on the range studied in this paper (Table 2). This result illustrates the importance of damping ratio on the impact forces. 
Table 2. Maximum $F_{m}$ in the smooth region for various damping

\begin{tabular}{ccccc}
\hline$\varepsilon$ & 0.1 & 0.2 & 0.3 & 0.5 \\
\hline$F_{m}$ & 3.75 & 2.46 & 1.45 & 0.93 \\
\hline$\frac{1}{2 \varepsilon}$ & 5 & 2.5 & 1.66 & 1 \\
\hline
\end{tabular}

\section{CHAOTIC REGION}

The bifurcation diagram of the first body in Fig. 13 illustrates the diversity of behaviour met by the nonlinear system. It switches from periodic solution to period-doubling bifurcation between $2.2<f_{r}<2.55$ and chaos between $2.55<f_{r}<2.7$. Then it returns to a periodic solution between $2.7<f_{r}<3.2$ and chaos 3.2 $<f_{r}<3.55$ and again a period-doubling bifurcation between $3.55<f_{r}<4.15$. Each change of regime is characterized by a significant modification of $F_{m}$.

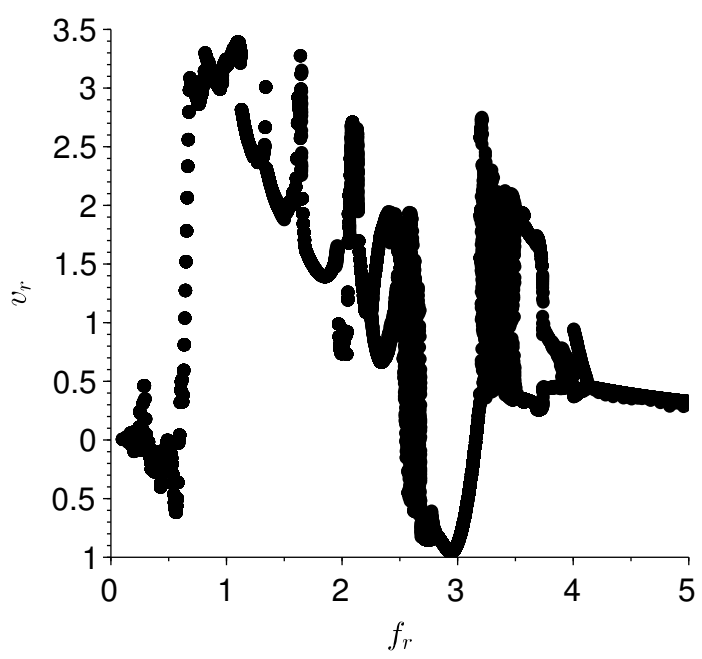

Fig. 13. Bifurcation diagram using $f_{r}$ as control parameter for $\mathrm{Nm}=5$ and $f_{c}=60$ and for $e_{r}=0.06$

Fig. 14 shows the maximal reduced impact forces for a small value of $e_{r}$ in the chaotic region. It can be observed that, unlike in the smooth region $F_{m}$ reaches its maximum values for various frequencies depending on $\mathrm{Nm}$ and $f_{c}$; this frequency increases with $f_{c}$ but decreases as $\mathrm{Nm}$ increases. Moreover, a very irregular evolution of $F_{m}$ as a function of the reduced frequency with abrupt decreases can be seen. The maximal impact forces obtain in the chaotic region are much more important than in the smooth region.

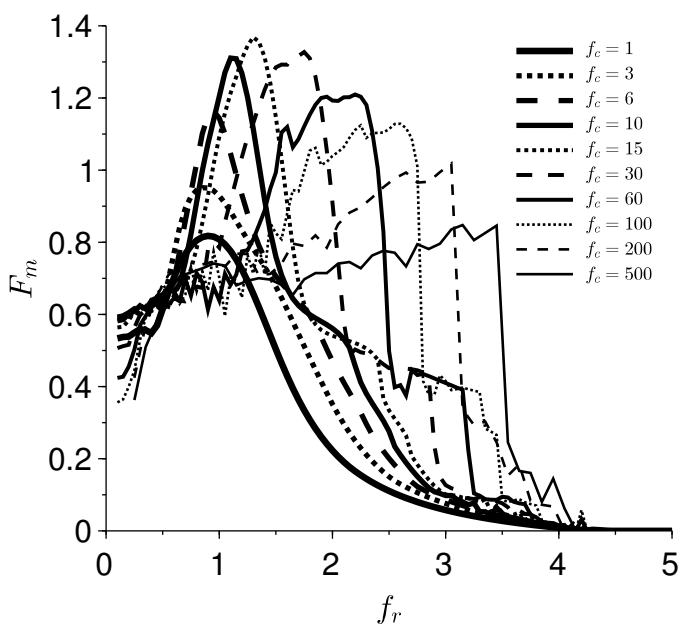

Fig. 14. Maximal reduced impact force as function of $f_{r}$ for $\mathrm{Nm}=5$ and various $f_{c}$, for $e_{r}=0.06$

The example of $F_{m}$ distribution in Fig. 15 shows that the forces may not be concentrated at the extremities and, in some cases, the maximum can be reached elsewhere.

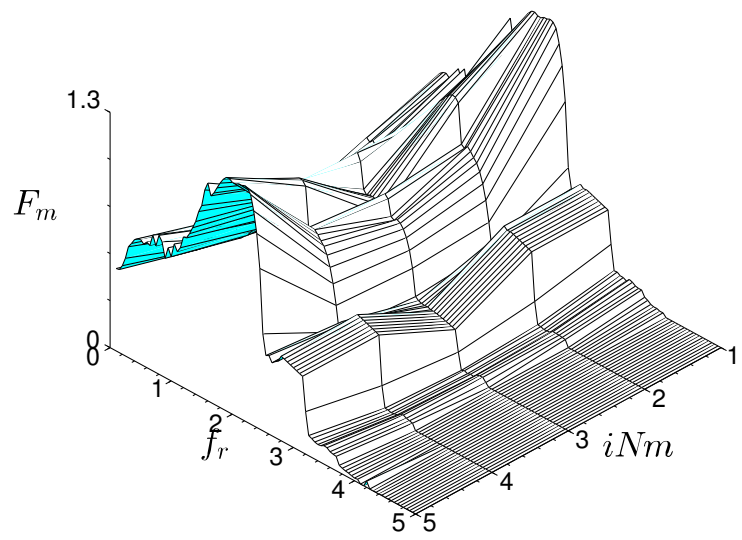

Fig. 15. Maximal reduced impact force spatial distribution as a function of $f_{r}$ for $N m=5, f_{c}=60$ and $e_{r}=0.06$ 


\section{LINEAR SYSTEM}

Let us consider the linear system without any gap. Since a simple analytical expression of the natural frequencies seems out of reach an approximation is proposed:

$$
\begin{aligned}
f_{k_{a}} & =\sqrt{1+f_{c}\left(\frac{k}{N m}\right)^{2}\left(a-b\left(\frac{k}{N m}\right)^{c}\right)} \\
a & =4.935 \\
b & =2.935 \\
c & =1.81 .
\end{aligned}
$$

This approximation gives a good estimation of the calculated natural frequency with an error of less than 1 $\%$ for $f_{1}$ and less than $3 \%$ for other natural frequencies on alarge range of values of $f_{c}$ and $\mathrm{Nm}$.

The approximated $f_{1}$ tend to 1 as $\mathrm{Nm}$ tends to infinity, and approximation gives the higher natural reduced frequency:

$$
f_{N m}=\sqrt{1+2 f_{c}}
$$

which is exact in the case of $N m=1$.

Let us define the reduced frequency:

$$
f_{r 1}=\frac{f_{r}}{f_{1_{a}}} .
$$

Plotting $F_{m}$ as a function of $f_{r 1}$ (Fig. 16) shows that all the curves reach their maximum values at the same reduced frequency. Thus, we can conclude from this observation that the maximum impact forces are obtained when the system behaves like a linear system oscillating at its first natural frequency. As observed previously, the impact forces increases when the damping ratio decreases (Fig. 17); moreover, for lower values of $\varepsilon$ the peaks due to second and third linear natural modes can be observed.

The maximal displacement of the first body on the first natural mode of the linear system is given by:

$$
u_{1 m}=\frac{1}{2 \varepsilon \sqrt{f_{1}^{2}-\varepsilon^{2}}} \Phi_{1}(1),
$$

where $\Phi_{1}$ is the shape of the first natural mode and given by the eigenvectors of $\mathbf{K}_{\mathbf{l}}$.

Let us assume that the first natural mode is close to a sinus distribution:

$$
\Phi_{1}(i) \approx \sin \left(\frac{\pi i}{N m}\right)
$$

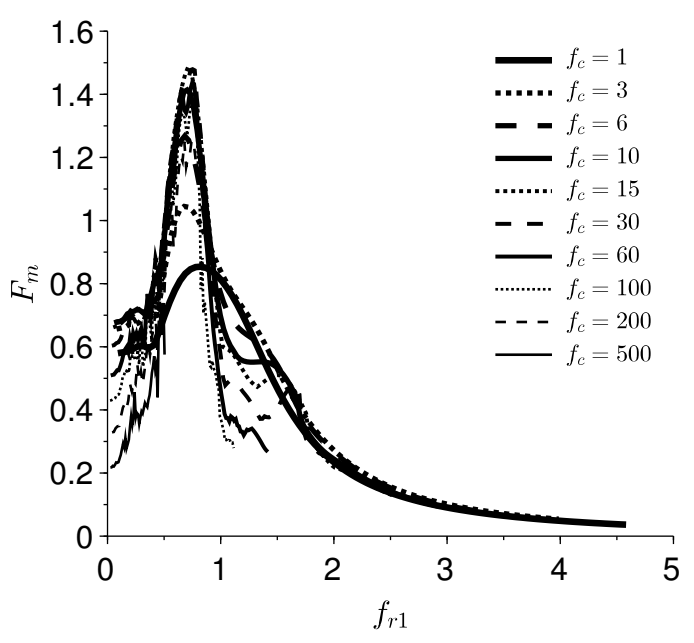

Fig. 16. Maximal reduced impact force as function of $f_{r 1}$ for $e_{r}=0$ and various $f_{c}$ for $\mathrm{Nm}=5$.

Assuming that $\mathrm{Nm}$ is high enough one can make the approximation:

$$
\Phi_{1}(1) \approx \frac{\pi}{N m} .
$$

Thus we can finally obtain an approximation of the maximal impact force:

$$
F_{m}=\sqrt{f_{c}} u_{1 m}
$$

$$
F_{m}=\frac{\pi}{2 \varepsilon N m} \sqrt{\frac{f_{c}}{1+\frac{f_{c}}{N m^{2}}\left(4.935-\frac{2.935}{N m^{1.81}}\right)-\varepsilon^{2}}},
$$

with an upper bound:

$$
F_{m}<\frac{\pi}{2 \varepsilon \sqrt{4.935}}
$$

Table 3 shows that the upper bound gives a good estimation of the maximal impact force but failes to give a conservative value.

Table 3. Maximum $F_{m}$ in the chaotic region for various damping

\begin{tabular}{ccccc}
\hline$\varepsilon$ & 0.1 & 0.2 & 0.3 & 0.5 \\
\hline$F_{m}$ & 7.02 & 3.88 & 2.65 & 1.86 \\
\hline$\frac{\pi}{2 \varepsilon \sqrt{4.935}}$ & 7.07 & 3.54 & 2.35 & 1.41 \\
\hline
\end{tabular}




\section{CONCLUSION}

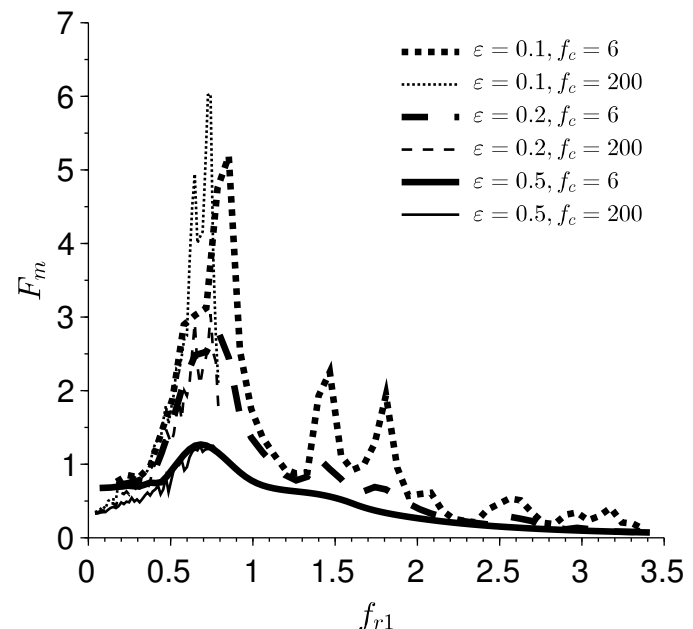

Fig. 17. Maximal reduced impact force as function of $f_{r 1}$ at $e_{r}=0$ for various $\varepsilon$, and for $N m=5$ and various $f_{c}$

\section{COMPARISON WITH EXPERIMENT}

The experiment described in [16] presents a row of 46 slender structures called fuel assemblies positioned vertically and clamped at their foot on a shaking table. The structures are likely to vibrate on their first natural bending mode, the clearance between structures is $3.5 \mathrm{~mm}$, and a seismic spectrum of about $10 \mathrm{~m} / \mathrm{s}^{2}$ is applied. The impact forces are measured; it has been shown that the maximal forces are located at the extremities as in the present study. Table 4 shows the dimensionless parameters of the experiment. It can be observed that the maximal reduced impact force encountered $F_{m}=0.52$ is much lower than the one estimated in the theoretical analysis $F_{m}=7.02$; thus, the simplified analysis seems to give a conservative upper bound. Nevertheless, this difference can be explained by the fact that each body is not a one-degree-of-freedom system, and some energy can be dissipated in higher modes, and the excitation in the experiment is not harmonic; thus, some transient effects are likely to occur.

Table 4. Experimental parameters

\begin{tabular}{ccccc}
\hline$\varepsilon$ & $N_{m}$ & $f_{c}$ & $e_{r}$ & $F_{m}$ \\
\hline 0.1 & 46 & 559 & 0.22 & 0.52 \\
\hline
\end{tabular}

The numerical results showed three regions. In the first one, there is no contact between the bodies. The second one defined as the smooth region is characterized by regular evolution with a concentration of the impact forces at the boundaries. In the last one, where the most important impact forces are met, the system shows chaotic behaviours, and coincide with the lower values of clearance. In the smooth region the criterion is respected, but in the chaotic region, some discrepancies are observed. Nevertheless, the criterion gives a good estimation. The dimensionless analysis showed that the maximal impact force is proportional to the mass, the acceleration, the stiffness ratio and the inverse of the damping ratio. Theoretical upper bounds for the maximal forces are proposed and compared to numerical and experimental results.

It would be interesting in further study to evaluate the effect of non homogeneous clearances and stiffness and nonlinear contact stiffness to be more representative of real life systems. In further studies a multi beam system will be studied and compared to the present results.

\section{NOMENCLATURE}

\begin{tabular}{|c|c|}
\hline$u_{i}$ & displacement, $[\mathrm{m}]$ \\
\hline$u_{e}$ & ground displacement, [m] \\
\hline$u_{d i}$ & relative displacement, $[\mathrm{m}]$ \\
\hline$M$ & mass, $[\mathrm{kg}]$ \\
\hline$C$ & damping, $\left[\mathrm{kg} . \mathrm{s}^{-1}\right]$ \\
\hline$K$ & stiffness, $\left[\mathrm{N} . \mathrm{m}^{-1}\right]$ \\
\hline$K_{c}$ & contact stiffness, $\left[\mathrm{N} \cdot \mathrm{m}^{-1}\right]$ \\
\hline$t$ & time, $[\mathrm{s}]$ \\
\hline$e$ & clearance, $[\mathrm{m}]$ \\
\hline$F_{r i}$ & right contact force, $[\mathrm{N}]$ \\
\hline$F_{l i}$ & left contact force, $[\mathrm{N}]$ \\
\hline$F_{r r i}$ & reduced right contact force, \\
\hline$F_{l r i}$ & reduced left contact force, \\
\hline$u_{r i}$ & reduced relative displacement, \\
\hline$f$ & ground frequency, [Hz] \\
\hline$f_{0}$ & natural frequency, $[\mathrm{Hz}]$ \\
\hline$a_{0}$ & ground acceleration amplitude, $\left[\mathrm{m} . \mathrm{s}^{-2}\right]$ \\
\hline$f_{r}$ & reduced frequency, \\
\hline$f_{c}$ & stiffness ratio, \\
\hline$\varepsilon$ & damping ratio, \\
\hline$t_{r}$ & reduced time, \\
\hline$e_{r}$ & reduced clearance, \\
\hline$N_{m}$ & number of bodies, \\
\hline$F_{m}$ & maximal reduced impact force. \\
\hline
\end{tabular}




\section{REFERENCES}

[1] Ricciardi, G., Bellizzi, S., Collard, B., Cochelin, B. (2009). Row of fuel assemblies analysis under seismic loading: Modelling and experimental validation. Nuclear Engineering and Design, vol. 239, no. 12, p. 2692-2704, DOI:10.1016/j.nucengdes.2009.08.029.

[2] Wei, D., Wang, Y., Jiang, T., Zheng, S., Zhao, W., Pan, Z. (2017). Chaos vibration of pinion and rack steering trapezoidal mechanism containing two clearances. Mechanical Systems and Signal Processing, vol. 92, p. 146-155,DOI:10.1016/j.ymssp.2017.01.019.

[3] Zhang, J., Guo, H-W., Liu, R-Q., Wu, J., Kou, Z-M., Deng, Z-Q. (2016). Nonlinear dynamic characteristic analysis of jointed beam with clearance. Acta Astronautica, vol. 129, p. 135-146, DOI:10.1016/j.actaastro.2016.09.009.

[4] Bai, Z.F., Sun, Y. (2016). A study on dynamics of planar multi-body mechanical systems with multiple revolute clearance joints. European Journal of Mechanics A/Solids, vol. 60, p. 95-111, DOI:10.1016/j.euromechsol.2016.06.009.

[5] Varney, P., Green, I. (2015). Nonlinear phenomena, bifurcations, and routes to chaos in an asymmetrically supported rotor-stator contact system. Journal of Sound and Vibration, vol. 336, p. 207-226, DOI:10.1016/j.jsv.2014.10.016.

[6] Li, Y., Chen, G., Sun, D., Gao, Y., Wang, K. (2016). Dynamic analysis and optimization design of a planar slider-crank mechanism with flexible components and two clearance joints. Mechanism and Machine Theory, vol. 99, p. 37-57, DOI:10.1016/j.mechmachtheory.2015.11.018.

[7] Rahmanian, S., Ghazavi, M.R. (2015). Bifurcation in planar slider-crank mechanism with revolute clearance joint. Mechanism and Machine Theory, vol. 91, p. 86-101, DOI:10.1016/j.mechmachtheory.2015.04.008.

[8] Luo, G.W., Lv, X.H., Shi, Y.Q. (2014). Vibro-impact dynamics of a two-degree-of freedom periodically-forced system with a clearance: Diversity and parameter matching of periodic-impact motions. International Journal of Non-Linear Mechanics, vol. 65, p. 173-195, DOI:10.1016/j.ijnonlinmec.2014.04.013.

[9] Wang, Z., Luo, T. (2017). Multiformity of periodic-impact motions of a harmonically forced soft-impacting system and experimental verification based on an electronic circuit. Chaos, Solitons and Fractals, vol. 94, p. 23-36, DOI:10.1016/j.chaos.2016.11.004.

[10] Liu, Y., Wang, Q., Xu, H. (2017). Analytical determination of bifurcations of periodic solution in three-degree-of-freedom vibro-impact systems with clearance. Chaos, Solitons and Fractals, vol. 99, p. 141-154, DOI:10.1016/j.chaos.2017.04.002.

[11] Liu, Y., Wang, Q., Xu, H. (2017). Bifurcations of periodic motion in a three-degree-of-freedom vibro-impact system with clearance. Communications in Nonlinear Science and Numerical Simulation, vol. 48, p. 1-17, DOI:10.1016/j.cnsns.2016.12.018.

[12] de Paula, A.S., Savi, M.A., Pereira-Pinto, F.H.I. (2006). Chaos and transient chaos in an experimental nonlinear pendulum. Journal of Sound and Vibration, vol. 294, no. 3, p. 585-595, DOI:10.1016/j.jsv.2005.11.015.

[13] Krasnopolskaya, T.S., Shvets, A.Y. (2009). Dynamical chaos for a limited power supply for fluid oscillations in cylindrical tanks. Journal of Sound and Vibration, vol. 322, no. 3, p. 532-553 DOI:10.1016/j.jsv.2008.09.007.

[14] Wei, D., Ruan, J., Zhu, W., Kang, Z. (2016). Properties of stability, bifurcation, and chaos of the tangential motion disk brake. Journal of Sound and Vibration, vol. 375, p. 353-365, DOI:10.1016/j.jsv.2016.04.022.

[15] Leng, X., Meng, G., Zhang, T., Fang, T. (2007). Bifurcation and chaos response of a cracked rotor with random disturbance. Journal of Sound and Vibration, vol. 299, no. 3, p. 621-632, DOI:10.1016/j.jsv.2006.07.006.

[16] Asayama, T., Kitamura, S., Morishita, M., Fontaine, B. (1999). JNC/CEA Collaborative work on core seismic study -SYMPHONY- Simulation of one-row mock-uo tests with restrained configuration. Transaction of the $15^{\text {th }}$ International Conference on Structural Mechanics in Reactor Technology, paper C03/01.

[17] Ladouceur, B., Woillez, J., Fontaine, M. (2004). Fuel assembly damping for accident studies: an analytical approach. Structural behaviour of fuel assemblies for water cooled reactors, Proceedings of a technical meeting, p. 271-278.

[18] Kerschen, G., Golinval, J.C., Vakakis, A., Bergman, L. (2005). The method of proper orthogonal decomposition for dynamical characterization and order reduction of mechanical systems: an overview. Nonlinear Dynamics, vol. 41, no. 1-3, p. 147-169, DOI:10.1007/s11071-005-2803-2.

[19] Graham, M.D., Kevrekidis, I.G. (1996). Alternative approaches to the Karhunen-Love decomposition for model reduction and data analysis. Computers Chemical Engineering, vol. 20, no. 5, p. 495-506, DOI:10.1016/0098-1354(95)00040-2

[20] Païdoussis, M.P., Sarkar, A., Semler, C. (2005). A horizontal fluid-conveying cantilever: spatial coherent structures, beam modes and jumps in stability diagram. Journal of Sound and Vibration, vol. 280, no. 1-2, p. 141-157, DOI:10.1016/j.jsv.2003.12.026.

[21] Bellizzi, S., Sampaio R. (2006). POMs analysis of randomly vibrating systems obtained from Karhunen-Loève expansion. Journal of Sound and Vibration, vol. 297, no. 3-5, p. 774-793, DOI:10.1016/j.jsv.2006.04.023. 